\title{
What is the Optimal Tool to Measure Gait Speed in a Clinical Setting?
}

\author{
Dae Young Kim ${ }^{1,2}$, Seung-Lyul $\mathrm{Oh}^{1}$ \\ ${ }^{1}$ Aging \& Mobility Biophysics Laboratory, Department of Rehabilitation Medicine, Seoul National University Bundang Hospital, Seongnam, Korea \\ ${ }^{2}$ Health and Exercise Science Laboratory, Department of Kinesiology, Seoul National University, Seoul, Korea
}

Physical changes due to age, cognitive decline, reduced agility to cope with the risk of falling, and environmental factors are becoming a significant threat to healthy aging. ${ }^{1)}$ In older adults, reduced gait speed is strongly associated with the risk of falls and limited physical functional capacity. ${ }^{2,3)}$ Gait speed measurement is the most important and simple test to assess changes in the physical function of older adults. ${ }^{4-6)}$ Gait speed is used not only to evaluate physical function but also to assess the general health status and diagnose sarcopenia in older adults. ${ }^{7)}$ Generally, manual stopwatch measurement is the most frequently used method to evaluate gait speed because it is easy, simple, fast, convenient, and economical and can be performed without the need for experts. ${ }^{8,9)}$ However, with the emerging importance of gait speed measurement, automatic sensors are increasingly used for more accurate measurement. $^{10)}$

We read the article by Jung et al. ${ }^{11)}$ with great interest. Our laboratory measures gait speed using automatic sensors and manual stopwatch. We agree with the results of the study performed by Jung et al. ${ }^{11)}$ in their cross-comparisons of gait speed measured using four different versions of automatic sensors and a conventional stopwatch. Until now, gait speed has mainly been measured manually using a stopwatch in clinical settings; however, gait speed assessment requires more accurate and consistent measurement for assessing the physical function in older adults. Therefore, cross-comparison of gait speed assessed by various automatic walking measurement equipment and stopwatch is an exciting and meaningful topic. In particular, we thank Jung et al. ${ }^{11)}$ for their impressive research on a more advanced version of the automatic sensor.

We previously compared the results of gait speed measured using automatic sensors by beam-breaking to those measured by manual stopwatch according to the starting protocols (standing start or moving start). ${ }^{12)}$ We suggested the need for careful attention to avoid misevaluation when gait speed was measured manu- ally using a stopwatch with a moving start. The use of automatic measuring equipment is recommended when a moving start is used as the starting protocol. Therefore, not only the timing method (manual stopwatch vs. automatic timer) but also the starting protocol (standing vs. moving start) require consideration in the study of gait speed measurement.

One disadvantage of measurement by the beam-breaking system described by the authors is an enlarged fanning effect in participants with wide-based gait or veering tendency. ${ }^{11)}$ To compensate for this problem, assessment of trunk movement with the sensor facing the participant's trunk rather than the side of the ankle has been proposed. However, this method requires some consideration. First, in the moving start method, the automatic measurement equipment is located in front of the end of walking, which may interfere with regular straight walking. Moreover, measurement of gait speed using longitudinal one-dimensional light detection and ranging technology requires a linear distance of about 10 $\mathrm{m}$ within the measurable range, imposing a space limitation in the clinical setting. The clinical utility of this equipment will be enhanced with troubleshooting of these issues in further studies.

\section{CONFLICT OF INTEREST DISCLOSURES}

The authors claim no conflicts of interest.

\section{REFERENCES}

1. Montero-Odasso M, Verghese J, Beauchet O, Hausdorff JM. Gait and cognition: a complementary approach to understanding brain function and the risk of falling. J Am Geriatr Soc 2012;60:2127-36.

2. Caetano MJ, Lord SR, Brodie MA, Schoene D, Pelicioni PH, Sturnieks DL, et al. Executive functioning, concern about falling 
and quadriceps strength mediate the relationship between impaired gait adaptability and fall risk in older people. Gait Posture 2018;59:188-92.

3. Brach JS, VanSwearingen JM, Newman AB, Kriska AM. Identifying early decline of physical function in community-dwelling older women: performance-based and self-report measures. Phys Ther 2002;82:320-8.

4. Graham JE, Ostir GV, Fisher SR, Ottenbacher KJ. Assessing walking speed in clinical research: a systematic review.J Eval Clin Pract 2008; 14:552-62.

5.Studenski S, Perera S, Wallace D, Chandler JM, Duncan PW, Rooney E, et al. Physical performance measures in the clinical setting. J Am Geriatr Soc 2003;51:314-22.

6. Lim J, Lim JY. Responsiveness of gait speed to physical exercise interventions in at-risk older adults: a systematic review and meta-analysis. Ann Geriatr Med Res 2017;21:17-23.

7. Cruz-Jentoft AJ, Bahat G, Bauer J, Boirie Y, Bruyere O, Cederholm T, et al. Sarcopenia: revised European consensus on definition and diagnosis. Age Ageing 2019;48:16-31.

8. Karpman C, Lebrasseur NK, Depew ZS, Novotny PJ, Benzo RP. Measuring gait speed in the out-patient clinic: methodology and feasibility. Respir Care 2014;59:531-7.

9. Maggio M, Ceda GP, Ticinesi A, De Vita F, Gelmini G, Costantino $\mathrm{C}$, et al. Instrumental and Non-Instrumental Evaluation of
4-Meter Walking Speed in Older Individuals. PLoS One 2016; 11:e0153583.

10. Kim M, Won CW. Combinations of gait speed testing protocols (automatic vs manual timer, dynamic vs static start) can significantly influence the prevalence of slowness: results from the Korean Frailty and Aging Cohort Study. Arch Gerontol Geriatr 2019;81:215-21.

11. Jung HW, Roh HC, Kim SW, Kim S, Kim M, Won CW. Crosscomparisons of gait speeds by automatic sensors and a stopwatch to provide converting formula between measuring modalities. Ann Geriatr Med Res 2019;23:71-6.

12. Oh SL, Kim DY, Bae JH, Jung H, Lim JY. Comparison of the use of a manual stopwatch and an automatic instrument for measuring 4-m gait speed at the usual walking pace with different starting protocols in older adults. Eur Geriatr Med 2019 [Epub]. https://doi.org/10.1007/s41999-019-00210-3.

Corresponding Author: Seung-Lyul Oh, $\mathrm{PhD}$

Aging \& Mobility Biophysics Laboratory, Department of Rehabilitation

Medicine, Seoul National University Bundang Hospital, 82 Gumi-ro

173beon-gil, Bundang-gu, Seongnam 13620, Korea

E-mail:oh3328@snu.ac.kr

ORCID: https://orcid.org/0000-0002-4730-6686

Received: September 13, 2019; Accepted: September 19, 2019 